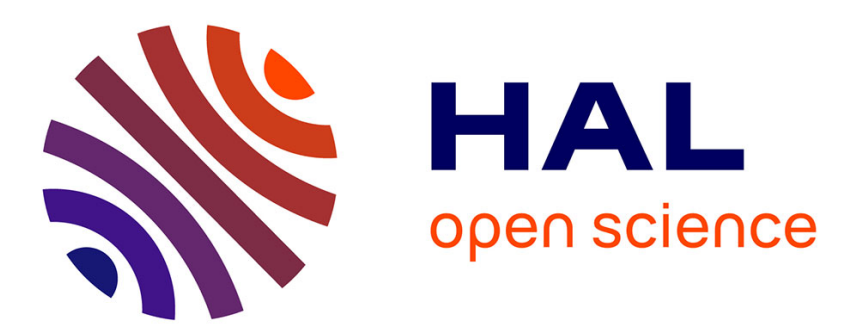

\title{
A Novel Multi-hop Body-to-Body Routing Protocol for Disaster and Emergency Networks.
}

\author{
Dhafer Ben Arbia, Muhammad Mahtab Alam, Rabah Attia, Elyes Ben \\ Hamida
}

\section{- To cite this version:}

Dhafer Ben Arbia, Muhammad Mahtab Alam, Rabah Attia, Elyes Ben Hamida. A Novel Multi-hop Body-to-Body Routing Protocol for Disaster and Emergency Networks.. WinCOM, Oct 2016, Fez, Morocco. hal-01649933

\section{HAL Id: hal-01649933 https://hal.science/hal-01649933}

Submitted on 28 Nov 2017

HAL is a multi-disciplinary open access archive for the deposit and dissemination of scientific research documents, whether they are published or not. The documents may come from teaching and research institutions in France or abroad, or from public or private research centers.
L'archive ouverte pluridisciplinaire HAL, est destinée au dépôt et à la diffusion de documents scientifiques de niveau recherche, publiés ou non, émanant des établissements d'enseignement et de recherche français ou étrangers, des laboratoires publics ou privés. 


\title{
A Novel Multi-hop Body-to-Body Routing Protocol for Disaster and Emergency Networks
}

\author{
Dhafer Ben Arbia*†, Muhammad Mahtab Alam*, Rabah Attia ${ }^{\dagger}$, Elyes Ben Hamida* \\ *Qatar Mobility Innovations Center (QMIC), Qatar University, Qatar Science and Technology Park (QSTP) \\ PO. Box. 210531, Doha, Qatar. Email: [dhafera, mahtaba, elyesb]@qmic.com, rabah.attia@enit.rnu.tn \\ ${ }^{\dagger}$ SERCOM Lab, Polytechnic School of Tunisia, University of Carthage B.P. 743. 2078 La Marsa. Tunisia
}

\begin{abstract}
In this paper, a new multi-hop routing protocol (called ORACE-Net) for disaster and emergency networks is proposed. The proposed hierarchical protocol creates an ad-hoc network through body-to-body (B2B) communication between rescue members and the command center. The on-body coordinators establish forward routes towards a command center, whereas, the command center builds reverse routes towards these nodes. The Routing tables are optimized based on real-time endto-end Link Quality Estimation (LQE) metrics (i.e., end-to-end signal strength level and end-to-end hop count). We evaluate our proposed protocol with other widely used protocols in the disaster context which are covered by the routing classes (i.e., Reactive, Proactive and Geographic-based). The evaluation is based on a realistic disaster mobility trace. The results show that the proposed protocol outperforms the other studied protocols in terms of packet reception rate and energy consumption. The proposed ORACE-Net protocol increases the body-to-body network lifetime and reliability.
\end{abstract}

Index Terms-Public Safety Networks (PSN), wireless Bodyto-Body Networks (BBN), Multi-Hop Routing Protocol, Performance Evaluation, WSNet simulator, Optimized Routing Approach for Critical and Emergency Networks Simulations.

\section{INTRODUCTION}

The worldwide climate change and the growing increase in disasters are not only impacting severely on human lives but also causing significant economic losses. According to the latest statistics from United Nations International Strategy for Disaster Reduction, 1.7 billion dollars were lost in disasters worldwide only in 2013 [1]. The role of emerging wireless communication technologies is critical because often during the disaster the existing network infrastructures are either completely damaged or over-saturated [2].

In Public Safety Networks (PSN), the data traffic is bidirectional which means the Command Center (CC) collects needed information from the deployed rescue teams and the rescue teams receive back real-time instructions to execute from their CC. From application perspectives, PSN may offer the possibility to transfer real-time video streaming and live data (e.g. physiological vital signs, position, orientation, mobility, etc.) through Internet. Thus, there are various purposes and requirements at the application layer.

Regarding the existing PSN, there are several challenges. In addition to the radio technologies and their inter-operability, coexistence and energy consumption issues, routing is also an important and critical challenge for the delay sensitive emergency networks [3]. For PSN, multi-hop communications is inevitable while routing the information from the incident area to the command center. In this context, it is expected that Wireless Body-to-Body Networks (BBNs) which is an evolution of traditional Body Area Networks(BAN), can act as an ad-on to the existing PSN networks [4], [5]. It creates an adhoc network which enables fast response and effective disaster management for monitoring the health conditions, movements, status of the rescue members and the victims [6].

Typically the existing routing techniques and protocols for PSN are limited by; a) Rescue members or deployed nodes are only able to transmit the information towards the command center node [7], whereas, bi-directional network connection is not supported; b) Inefficient beacon enabled and broadcast of hello packets to discover and update the neighbor nodes; c) Inability to communicate with the standard networks (e.g. Internet), in addition to limited services being provided (i.e., emergency messaging and nodes status sharing [7]); d) MANET routing protocols (which are often used in PSN) consider the entire network as flat (i.e., all the nodes of the network have the same level of privilege), while disaster networks need to be hierarchically organized (logically or operationally) in order to the communication based on its priority.

In this paper we propose a new hierarchical multi-hop bodyto-body optimized routing approach targeted for critical and emergency networks (ORACE-Net) with an aim to improve the connectivity of the multi-hop body-to-body routing for PSN. Specific contributions are explained as follows. In contrast with existing routing protocols, ORACE-Net relies on the broadcast of advertisement (ADV) packets (for neighborhood discovery) which avoids the transmission of control overheads such as route request and route reply messages. At first by using the ADV packets, the protocol establishes forwarddirection communication paths from the entire network to the command center, and then we introduce reverse routing path establishment for bi-directional routes (i.e., from the command center to the rest of the network) based on the data packets transmission. This helps in optimizing the overall communication overheads and increases the network performance. The proposed protocol continuously update the routes based on the link quality estimation (i.e., received signal strength) and the reliable shortest path estimates in a mobile environment. Finally, packet oriented network simulator is exploited for extensive performance evaluations and comparisons. The 
simulations are performed under realistic assumptions with the disaster mobility models. The obtained results show that ORACE-Net outperforms the conventional routing approaches both in terms of packet reception rate and energy consumption.

Specific contributions of this paper are listed as follows: 1) We present ORACE-Net approach which is based on the Endto-End Signal Strength Level $\left(E 2 E_{S S L}\right)$ to optimize routing in disaster networks. 2) We simulate and evaluate the protocol in realistic disaster scenario and scheme: dimensions of the area, disaster mobility pattern, MAC and PHY layers (pathloss, TX-power, modulation, etc.). 3) We compare our protocol behavior to eligible routing candidates from each routing class (proactive, reactive and geographic-based).

\section{RELATED WORKS}

With regards to routing the information in emergency and public safety networks, there is hardly any dedicated routing standards. Recent works are mainly focused on three main approaches: a) To investigate the existing standards behaviors in the disaster and emergency context [8], [3]; b) To enhance the existing standards to fit disaster networks requirements [9], [10]; c) To propose new approach and compare it to existing standards to evaluate their performance [7], [11]. However, these new approaches are very basic and limited in terms of real disaster characteristics.

Latest research trends are tending towards evaluating the performance of enhanced existing conventional routing protocols. However, some recent studies (such as [7], [10][13]) are adapted to the network dynamic aspects for viable and effective disaster operation. For example, localizationbased and network congestion adaptive approach called "DistressNet" [12] is very interesting to avoid congestion in the network during disaster operations. However, this approach creates network sparseness which impacts localization and renders multi-hop algorithms inefficient especially in the indoor rescue operations. Further, recent proposed approaches such as Reliable Routing Technique (RRT) [11] and TeamPhone [7], on principal, are based on Greedy Perimeter Stateless Routing (GPSR) [14] and Delay Tolerant Network version2 (DTN2) [13] concepts respectively. The authors claim that RRT approach is better in terms of delay, however, it has significantly higher energy consumption because of unnecessary broadcast packets. On the other hand, TeamPhone is limited in terms of service, in fact it considers only basic emergency messages with up to only one hop neighbors based on ad-hoc and opportunistic networks. Some other varieties of DTN protocols are efficient in terms of packet delivery rate, however, delay is a big drawback that makes DTNs not suited for our context. Finally, the concept of multihop smart-phone networks based on WiFi-Direct is proposed in [10]. An energy efficient cluster-based routing protocol, called Quasi Group Routing Protocol is used. In addition, virtual hierarchical distributed cluster algorithm for smartphone networks is introduced. The simulations demonstrate that the proposed protocol can save significant amount of energy. However, the proposed approach is limited to a basic simulation environment without considering the impact of the mobility.
With regards to the behavior of the existing standards which were designed for general purposes, such as Mobile Ad-hoc Networks (MANET), Geographical Location based, or Wireless Sensor Networks (WSN) routing protocols [3]. Typically, proactive and reactive multipath routing Protocols are commonly referred as the link-state and distance vector algorithms, hence their classification is based on network discovery and the routing information update mechanism [15]. Proactive routing protocols create and maintain continuously their routing tables and are also called table-driven. In this routing class, nodes keep exchanging information to learn the network topology. The most widely known proactive routing protocols include Optimized Link State Routing protocol (OLSR) [16], Wireless Routing Protocol (WRP), DestinationSequenced Distance-Vector (DSDV) and recently the OLSR version 2. In PSN, such routes discovery techniques and computation cause network overloading that impacts the bandwidth utilization and increases the power consumption. Drawbacks of proactive routing protocols in PSN are not only due to energy inefficiency and bandwidth overload, but also the routing convergence delay caused by the intensive routes discovery broadcasts at the network start-up is also considerable.

Unlike proactive protocols, reactive routing protocols look up for routes only when it is needed. The route discovery procedure is invoked when data packets are ready to send. The route discovery mechanism in reactive routing protocols is the same i.e., a source node starts by flooding a request message to all reachable nodes looking up for the destination, then, each node relays this request message until it reaches the destination. If the destination is reached, a reply message is sent back to the source node through the reverse route followed by the request. Most known on-demand routing protocols are Ad-hoc On demand Distance Vector (AODV) [17], Dynamic Source Routing protocol (DSR), Temporally Ordered Routing Algorithm (TORA) and more recently AODV version 2 (called also DYMO). Reactive routing protocols in mobile ad-hoc networks seem to solve the bandwidth utilization and energy efficiency issues based on the on-demand routing request, but the delay caused by the route discovery before data transfer, does not meet the PSN requirements. In addition to Ad-hoc routing protocol classes mentioned above, hybrid routing protocols class merge both reactive and proactive routing techniques. These routing protocols offer generally a combination between proactive routing for nearby nodes and reactive routing protocols for faraway nodes. Some other protocols are 3GPP based and are out of our related works scope since our proposed protocol is WiFi based. These approaches may be investigated in our upcoming studies and among the experimentation.

To summarize, multi-hop body-to-body communication is incapable to connect the whole disaster network. In our latest findings in [3], it is found necessary to build a bi-directional routes between the PSN command centers and the deployed rescue members in emergency and disaster situations. However, to the best of our knowledge, the aforementioned routing protocols are not optimized for the PSN context and have many limitations. While comparing the studied routing protocols both MANET and PSN, as illustrated in Table I, our proposed 
TABLE I

Routing Protocols Benchmark.

Specifications

\begin{tabular}{cc|cccccc}
\hline \multicolumn{2}{c}{ Routing Protocols } & $\begin{array}{c}\text { Routing } \\
\text { Strategy }\end{array}$ & $\begin{array}{c}\text { PSN- } \\
\text { Architecture }\end{array}$ & Scalability & Delay & PRR & $\begin{array}{c}\text { Energy- } \\
\text { Efficiency }\end{array}$ \\
\hline \multirow{2}{*}{ MANET } & $\begin{array}{c}\text { AODVv2 } \\
\text { OLSRv2 }\end{array}$ & $\begin{array}{c}\text { Reactive } \\
\text { Proactive } \\
\text { Geographic- } \\
\text { Based }\end{array}$ & $\begin{array}{c}\text { Flat } \\
\text { Flat }\end{array}$ & $\begin{array}{c}\text { Scalable } \\
\text { Scalable } \\
\text { Depends on } \\
\text { Positioning } \\
\text { system }\end{array}$ & $\begin{array}{c}\text { High } \\
\text { Low } \\
\text { Medium-to- } \\
\text { High }\end{array}$ & $\begin{array}{c}\text { Low } \\
\text { Medium } \\
\text { Low-to- } \\
\text { Medium }\end{array}$ & How \\
Low \\
PSN
\end{tabular}

approach is optimized based on the following features: 1) With reference to AODVv2 and OLSRv2, it is optimized because it only uses one type of packets (i.e., ADV) in both neighbor discovery, link quality estimations and direct route establishment (i.e., from the deployed nodes towards the CC-node). This strategy decreases significantly the energy consumption and the network congestion as can be seen from the simulations results in Section V. 2) In addition, the proposed protocol relies on the data packets to establish reverse routes (i.e., from the $\mathrm{CC}$-node towards the deployed nodes) which is typically not considered in disaster and emergency networks. 3) Routes established by our proposed protocol are continuously updated according to the received signal strength level and the hop count. Consequently, builds connectivityaware routing paths. 4) In contrast to OLSRv2 (which relies on the concept of Multi-Point Relays (MPRs) to control the topology), our approach is an optimized proactive protocol, it does not elect any special node (i.e., MPR), it is hierarchically designed with regards to the critical and emergency network architecture (Command Center and simple deployed nodes). Finally, PSN-supported routing protocols are very basic with limited features as mentioned above. The proposed ORACENet protocol is targeted for disaster and emergency network characteristics, with an aim to improve the connectivity of the existing PSN.

\section{Multi-hop Body-TO-Body Optimized Routing APPROACH FOR CRITICAL AND EMERGENCY NETWORKS (ORACE-NET)}

In this section we present the newly proposed routing protocol (called ORACE-Net: Optimized Routing Approach for Critical and Emergency Networks) with the main objective to have instant neighborhood links visibility and available optimized routes according to the specific link quality estimation metrics. The new proposed routing protocol consists into three main phases. First, Advertisement broadcasts, second, Direct Route Establishment (DRE) and third, Reverse Route Establishment (RRE).

1) Advertisement broadcasts and Link Quality Estimation: The Command Center node (CC-node) initializes the connection by broadcasting periodically Advertisement packets
$(A D V)$ which are flooded over the entire network as a wave to introduce the $C C$-node to the rest of the nodes in the network. A node receiving an $A D V$ packet, rebroadcasts it to all its reachable neighbors. The header of the $A D V$ packet contains a sequence number used to discard the duplicated received $A D V$ packets. The last visited node is also recorded into the $A D V$ packet header and then utilized as the next-hop in the established routes towards the $C C$-node.

In our proposed approach, $A D V$ broadcasting process has three key roles. First, it replaces conventional neighbors discovery process, since all neighbors rebroadcast the received $A D V$ packets, so a classical Hello protocol is not needed anymore. The $A D V$ communicates to the network the $C C$ node $(S)$ information and provides the neighborhood discovery. Second, based on the $A D V$ packets (flooded within a period of 3 seconds) nodes establish their routes towards the $C C$ node $(S)$. Third, it provides the Signal Strength Level between two nodes. The new proposal relies on two main metrics: the cumulative end-to-end links quality estimation (LQE) and the end-to-end hop count. The first metric might be calculated based on the Signal Strength Level (SSL), the link quality indicator (LQI) or the signal to noise ratio (SNR) measurement [18]. To that end, each $A D V$ packet contains specific header's entries to track the hop count and the cumulative LQE (noted as $E 2 E_{S S L}$ ) along the traversed route. When an $A D V$ packet is rebroadcast, the $E 2 E_{S S L}$ field in the packet header is updated by summing the $S S L$ values recorded at each hop as depicted in Figure 1, according to the following equation:

$$
E 2 E_{S S L}^{S D}=\sum_{S}^{D} S S L_{i j}
$$

where: $S$ is the source of the E2E route, $D$ is the destination, $i$ and $j$ are the visited nodes from the source to the destination. $S S L_{i j}$ is the received signal strength between node $i$ and $j$ (i.e., on one hop only).

In the remaining sections of the paper we consider the Endto-End Link Quality Estimation $\left(E 2 E_{L Q E}\right)$ as the End-to-End Signal Strength Level $E 2 E_{S S L}$. 


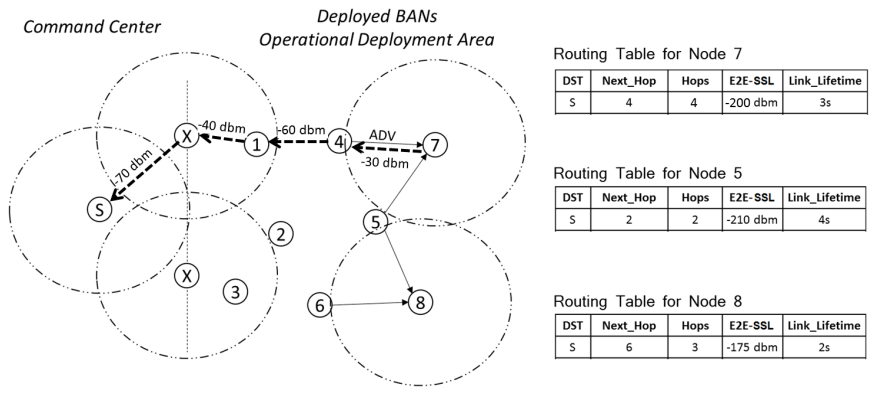

Fig. 1. Routing tables (for Nodes 7, 5 and 8) when the $1^{\text {st }}$ wave of ADV reaches all nodes. Nodes $\mathrm{X}$ are base stations deployed by the rescue teams while they are moving towards the incident area.

As an example, $E 2 E_{S S L}$ of the route from node 7 towards the $C C$-node $(S)$ is calculated based on 1 and as follows:

$$
\begin{aligned}
E 2 E_{S S L}=(-30 \mathrm{dbm})+ & (-60 \mathrm{dbm})+(-40 \mathrm{dbm}) \\
+ & (-70 \mathrm{dbm})=-200 \mathrm{dbm}
\end{aligned}
$$

2) Direct Route Establishment (ADV-Based): The CC-node broadcasts periodically $A D V$ packets with incremented sequence number, when the $A D V$ packet is received by a neighbor node, that node updates both its neighbors and routing tables, then it rebroadcasts the $A D V$ again. The $A D V$ packet header contains the $E 2 E_{S S L}$ and the Hop Count. Once received by a node, first step is updating the neighbors table. Second, the E2E $E_{S S L}$ and the Hop Count fields are extracted and their values are compared to those recorded in the current route towards $C C$-node. If the new values of $E 2 E_{S S L}$ and the $H$ op $_{\text {Count }}$ offer better optimized route, then the routing table is updated, otherwise, the $A D V$ packet is rebroadcast without any change. These steps are depicted in the Algorithm 1.

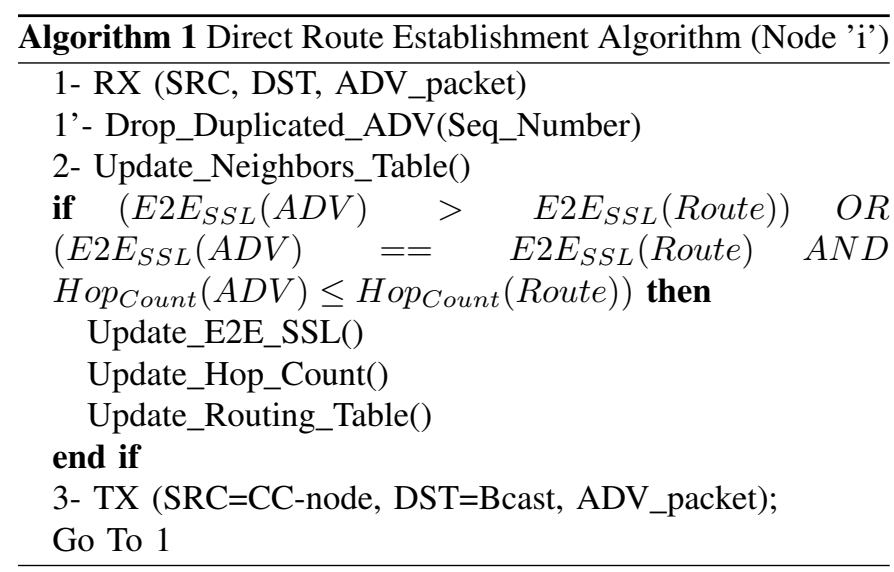

According to algorithm 1, each node selects the best route based on the highest end to end Signal Strength Level value and in case of equality the algorithm chooses the shortest path. In the case where $E 2 E_{S S L}(A D V)$ is lower than $E 2 E_{S S L}$ (Route), the node waits until the lifetime (3 times periods of $A D V$ ) of the route is expired, then, the sender of the first received $A D V$ is selected as next-hop and the DRE process is relaunched again.

The Direct Route Establishment phase of the protocol ends up by a routing table at every reachable node with only one way routes (i.e., from nodes towards the $C C$-node). Once a route is established towards the $C C$-node, nodes send back a data packet to the $C C$-node, this data packet has a key role for the next phase called Reverse Route Establishment (i.e., $R R E)$.

3) Reverse Route Establishment(Data-based): Based on the previous phases (i.e., $A D V$ broadcast and DRE), each node of the network is able to establish an optimized route towards the $C C$-node and to estimate the $E 2 E_{S S L}$ and the hop - count accordingly. As a reply to the $A D V$ packet, nodes start sending back data packets to the $C C$-node. The main challenge raised by our proposed approach is to establish the reverse way routes: Routes from the $C C$-node( $S$ ) to the rest of the nodes in the network. Indeed, the data packets are forwarded hopby-hop until they reach the $C C$-node. When a node receives a data packet with the $C C$-node as a final destination node, the following fields are extracted from the data packet header: $E 2 E_{S S L}$, the hop-count, and the last visited node. A new (or existing) route is created (or updated) with the gathered values from the extracted fields of the data packet to fill respectively the following entries in the route: $E 2 E_{S S L}$, the hop-count, and next-hop. Figure 2 illustrates the data packets flow towards the $C C$-node and the final routing table established by the $C C$-node. Once a data packet reaches the $C C$-node, an entire route is established towards the source of the data packet. Similarly to the DRE phase, the $E 2 E_{S S L}$ metric is calculated and updated using the same process and equation 1 .

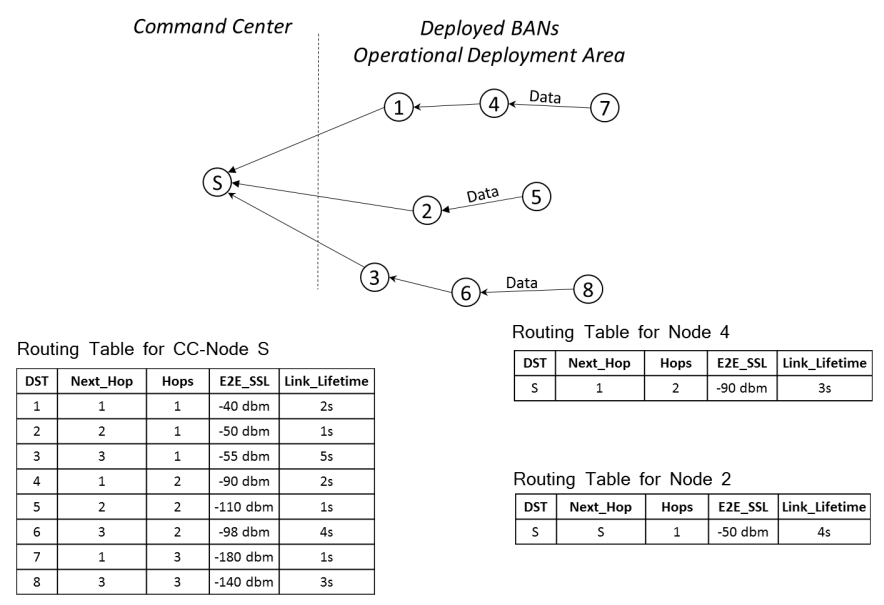

Fig. 2. Reverse Route Establishment (i.e., RRE) based on data packets.

\section{DisASTER SCEnARIO}

In this paper we investigate a disaster scenario (fire trigger as a case study) in the "Landmark" shopping mall in the State of Qatar as depicted in figure $3(500 / 300 \mathrm{~m})$. The mobility model used is generated by the BonnMotion tool [19]. Bonnmotion generates a mobility trace file which is used later in the packet oriented network simulator WSNET [20]. The total number of rescuers (i.e., firefighters, medical teams, helping civilians) is equal to 100 . We assume that the fire is triggered into two opposite sides of the mall (i.e.,incidents areas), and the speed of the rescuers is less than $1.3 \mathrm{~m} / \mathrm{s}$. 


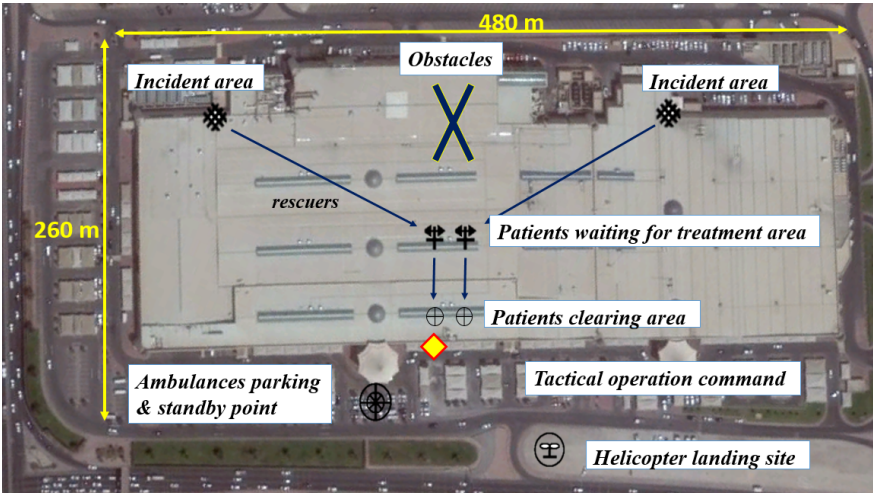

Fig. 3. Disaster Area Nodes Locations, Areas and Obstacles.

Rescuers, considered as mobile nodes, follow the disaster mobility model generated by Bonnmotion. Bonnmotion disaster mobility scheme is detailed in [21].

Figure 3 shows the different areas of the disaster scenario generated by Bonnmotion, where nodes (rescuers) are saving and transporting victims from the incident areas to a safe place (patients waiting for treatment area and patients clearing area). The entire nodes inside the mall consist of an ad hoc network, where the CC-node is placed at the main-gate of the mall (shown as ' $\diamond$ ' symbol in figure 3 ). Nodes send their data (i.e., images, videos, medical status) towards the $C C$-node, where a command center is conducting the operations.

\section{Performance Evaluation}

In this section we present the network level performance evaluation and simulations results of the proposed routing protocol. For comparison, with the respect to each relevant class of routing protocols (i.e., Reactive routing, Proactive routing, and Geographic-based routing) we selected the pertinent standard or protocol. The eligible selected candidates to investigate and compare are the most widely used routing protocols (i.e., AODVv2, OLSRv2 and GPSR). It is crucial to reproduce the realistic critical and disaster relief environment for the realistic performance evaluation. Therefore, several essential factors must be considered. First, a realistic mobility model (spatial and temporal consideration, mobility pattern, unpredictable crowd behavior, multiple incidents areas, etc.). Second, command and control operational requirements (e.g., Command Center location, rescue groups formation, logistic and medical resources, etc.). Third factor, simulated dynamic information generation (data flow in two ways: from the Command Center to the incident area and vice versa). In fact, first and second factors are related to the mobility modeling tool features, whereas, third factor depends on the network simulator used (detailed in the following sub-section i.e., V-A).

\section{A. Simulation Setup and Mobility Modeling}

The mobility scenario generation and analysis tool called Bonnmotion implements widely used existing models [19]. The Disaster Area Mobility model proposed in Bonnmotion is designed and implemented specifically for the crisis and
TABLE II

Simulation Setup Parameters - WSNET v3.0

\begin{tabular}{|l|l|}
\hline Parameters & Values \\
\hline \hline Area & $500 \mathrm{~m} / 300 \mathrm{~m}$ \\
\hline Number of nodes & 100 \\
\hline Number of CC-nodes & $\begin{array}{l}1 \text { (located in the left corner of the area with the } \\
\text { coordinates }[0,250,0] \text { ) }\end{array}$ \\
\hline Simulation Duration & 300s/iteration \\
\hline Mobility Modeling & Disaster Area Scenario \\
\hline Application Layer & $\begin{array}{l}\text { Constant Bit Rate(CBR) with 1 packet///node } \\
\text { Payloads : 200, 400, 600, 800 and } 1000 \text { bytes }\end{array}$ \\
\hline Routing Layer & AODVv2, OLSRv2, GPSR and ORACE-Net \\
\hline MAC-Layer Standard & IEEE802.11b CSMA/CA DCF with ACK \\
\hline PHY \& Radio-Layer & $\begin{array}{l}\text { Pathloss = freespace, Modulation }=\text { BPSK, } \\
\text { Sensitivity = -92dBM, TX Power = 0dBm, } \\
2.4 \mathrm{GHz}\end{array}$ \\
\hline Packets Sizes & According to the protocols standards [16][17][14] \\
\hline Number of iterations & 15 per protocol per payload \\
\hline
\end{tabular}

public safety context (e.g., Earthquake, Air crash, storm, tsunami). The disaster scenario mobility model is the most appropriate model to use in case of critical and emergency simulation [3]. Indeed, this model provides a realistic pattern of real disaster theater composed of incident area(s), casualties clearing area(s), transport and medical evacuation zone(s).

The simulation setup and respective parameters are detailed in Table II as follows: The mobility trace file is generated according to the disaster mobility model. For systems level simulations, we are using an event-driven, packet-oriented network simulator called WSNet (version 3.0) [20]. Once, the mobility trace file is converted into the input file format of WSNET, we start the simulations in the following order. We select one of the routing protocols (i.e., ORACE-Net, AODVv2, OLSRv2 or GPSR), then, an application layer payload is selected (from: 200, 400, 600, 800 or 1000 bytes per second (Bps)). We are considering these payloads for simulation only, without consideration of big overhead in the applications layer for all the protocols. We are considering a free-space path-loss because the mobility model considers obstacles inside the disaster area where nodes can not cross and have to go around them. Indeed, a free-space path-loss would be fair for the model with an approximate range of 50 meters (based on the TX-power). We ran 15 iterations with the combination "Protocol-Payload", when done, we select the next payload value with the same routing protocol, once finished, we select the next routing protocol. We run the simulation with $95 \%$ of confidence interval considered. Once a specific routing protocol simulation is completed with all the payload values for the considered iterations, the next routing protocol is selected, and so on. This process is coordinated by shell scripts that select, run and save the results.

\section{B. Simulations Results}

The performance metrics considered for the evaluation of the protocols are: First, the Average Packet Reception Rate (i.e., PRR) which consists of the number of received packet divided by the number of the transmitted packets at the application layer. Second, the Average Communication Delay, which is the average packet delay between the source node 


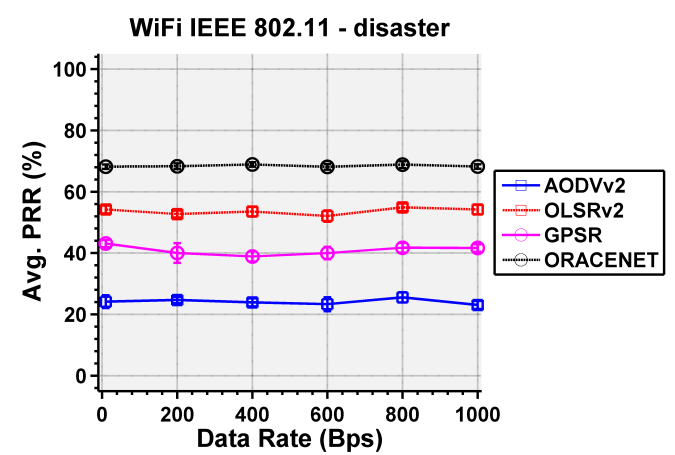

(a)

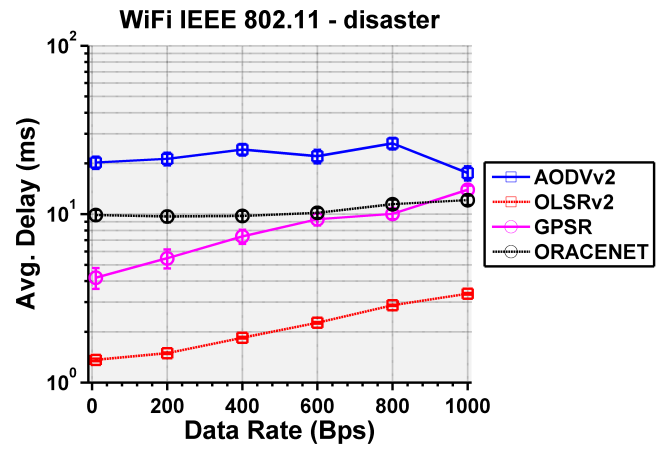

(c)

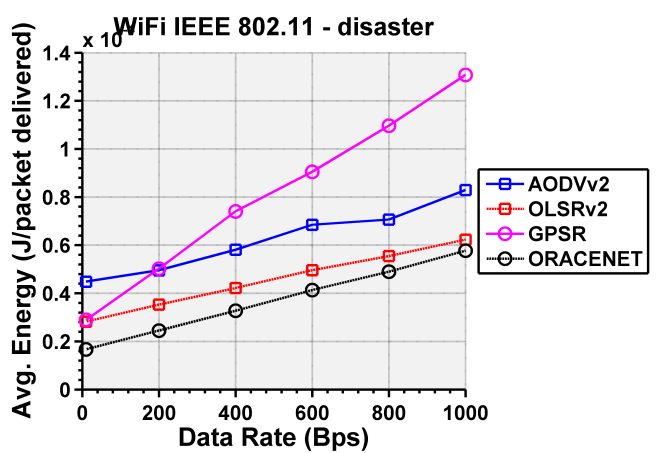

(b)

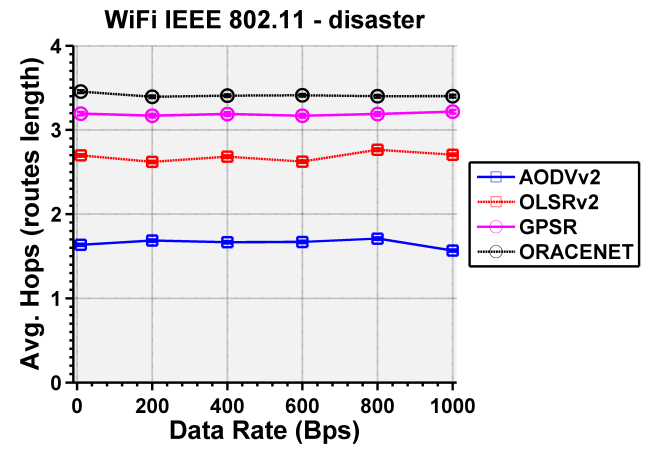

(d)

Fig. 4. Multi-hop Body-to-Body Routing Protocol Behavior with Disaster Scenario mobility model.

and the final destination over a multi-hop communication. Third, the Average Hop Count, which consists of the average number of hops starting from the source to the destination in the network. Finally, the Average Energy Consumption per delivered packet with the considered radio transceiver parameters detailed in Table II. It is important to note here that the energy efficiency is an important challenge in the disaster context. Indeed, a rescue team may be working along few hours to more than one day.

a) Packet Reception Rate: Figure 4-(a) shows the average PRR for the different studied protocols. Our proposed protocol (ORACE-Net) achieves the best performance with nearly $70 \%$ of PRR while OLSRv2 has a difference of more than $10 \%$ of PRR lower than our approach. On the other hand, AODVv2, achieves the worst performance with $23 \%$ of PRR mainly due to the high number of re-transmissions and the non-resolved destinations. Indeed, a route in AODVv2 is established on-demand, when the route is set, and the nodes have a high mobility behavior (which is the case here), the route must be updated continuously, if AODVv2 keeps on using an old route where intermediate nodes moved away, that route is no more available, a Route Error (i.e., RERR) is received by the sender, and a new request is flooded into the network. All this process has an impact on the average PRR, communication delay and the energy consumption. GPSR has an average PRR around $40 \%$ with the different payloads. Obviously, GPSR may have better performance in outdoor scenario, an indoor GPS location system is not efficient, and has a significant impact on the energy consumption.

b) Energy Consumption: The energy consumption with a disaster scenario is shown in the Figure 4-(b). The energy consumption for each transmitted packet is calculated as follows,

$$
E_{\text {Packet }}=T_{\text {Packet }} \times 3_{\text {Volts }} \times I_{m A}
$$

where, $T_{\text {packet }}$ is the duration in milliseconds which is based on the effective packet length (including all the PHY and MAC headers [22]).

GPSR has increasingly higher energy consumption starting from 200Bps payload, this is due to its considerable energy consumption of the GPS receiver. Our proposed approach performs much better compared to the other protocols mainly because of having lower network control packets (i.e., RREQ, RREP, REER, Topology Control) and the non-use of periodic beacons which reduces the energy consumption. The behavior of AODVv2 and OLSRv2 are quite comparable, but according to the average PRR results, AODVv2 is not resolving the routes since it has lowest PRR. Thus, AODVv2 is consuming this amount of energy in sending the Route Error and the new Route Request packets. Overall, the energy consumption of all the routing protocols is increasing accordingly with the payloads.

c) Communication Delay: Figure 4-(c) depicts the average communication delay for the four studied routing protocols with a Disaster Area mobility model. It can be seen that AODVv2 has the highest and variable results. The unstable behavior of AODVv2 is due to the high topology change which ensues in low average PRR recorded (as explained above) and high average delay. However, OLSRv2 and GPSR have better performance in terms of delay accordingly with the low PRR. Indeed, the PRR of OLSRv2 and GPSR is low, so there is no much data delivered and there will be lower communication delay. On the other hand ORACE-Net records a higher average delay than OLSRv2 and GPSR which is related with the higher average PRR. Moreover, the quantity of processed data packets 
with the ORACE-Net is much higher than the rest of the protocols. ORACE-Net behavior in terms of delay is fair with reference to the number of delivered packets.

d) Average Hop Count: Figure 4-(d) shows the average hop count with a disaster mobility scenario. All the routing protocols except AODVv2 have approximately the same performance (i.e., between 2.7 and 3.5 hops) in terms average hop count. ORACE-Net has the highest hop count (3.5 hops). However, based on the average PRR and energy, the established routes have higher hop count but they are more reliable than those established by the other protocols (i.e., AODVv2) despite they are shorter.

Finally, the proposed protocol is the most performant routing protocol simulated with realistic disaster mobility scenario. OLSRv2 is close to ORACE-Net in terms of energy consumption and average hop count, but far in terms of PRR. Whereas, it is concluded that AODVv2 and GPSR are not suitable to be deployed in disaster mobility network. To summarize, for higher connectivity in a mobile disaster scenario, the proposed ORACE-Net is the most appropriate routing protocol.

\section{CONCLUSION AND FUtURE WORKS}

In this work, we have presented a new optimized routing approach for critical and emergency networks ORACE-Net). Based on advertisement packets to establish routes from deployed nodes towards the Command Center node, we call this process Direct Route Establishment (DRE). DRE is followed by the Reverse Route Establishment (RRE) step, which allows the $C C$-node to build routes towards all the nodes of the network. Our proposed protocol has been evaluated based on realistic disaster mobility model with an event-driven simulator (i.e, WSNET). Compared to eligible selected standards and protocols from each routing class (i.e., AODVv2 as reactive, OLSRv2 as proactive and GPSR as geographic-based routing protocols), our approach shows best performance compared to the other protocols in terms of average PRR. Additionally, the proposed approach is energy efficient. To conclude, the new proposed approach increases the network lifetime and optimizes the routes based on an End-to-End Signal Strength Level with minimum hop count. ORACE-Net will be implemented to experiment the protocol in real conditions and investigate its behavior to fulfill the application layer requirements (i.e, Internet connection, medical services, vital monitoring), and this will be covered in the future works.

\section{ACKNOWLEDGMENT}

This publication was made possible by NPRP grant \# $[6-$ $1508-2-616]$ from the Qatar National Research Fund (a member of Qatar Foundation). The statements made herein are solely the responsibility of the authors.

\section{REFERENCES}

[1] UNISDR. (2013) Global assessment report on disaster risk reduction 2013. [Online]. Available: http://www.preventionweb.net/english/hyogo/ gar/2013/en/home/download.html

[2] G. Baldini, S. Karanasios, D. Allen, and F. Vergari, "Survey of wireless communication technologies for public safety," IEEE Communications Surveys Tutorials, vol. 16, no. 2, pp. 619-641, Second 2014.
[3] D. B. Arbia, M. M. Alam, R. Attia, and E. B. Hamida, "Behavior of wireless body-to-body networks routing strategies for public protection and disaster relief," in proceedings of the Workshop on Emergency Networks for Public Protection and Disaster Relief (EN4PPDR 2015), in 11th IEEE WiMob Conference, Oct. 2015.

[4] E. B. Hamida, M. M. Alam, M. Maman, B. Denis, and R. D’Errico, "Wearable body-to-body networks for critical and rescue operations, the crow ${ }^{2}$ Project," in procedding of the Workshop on the Convergence of Wireless Technologies for Personalized Healthcare in IEEE PIMRC 2014, Sept. 2014, pp. 2145-2149.

[5] M. M. Alam and E. B. Hamida, "Surveying wearable human assistive technology for life and safety critical applications: Standards, challenges and opportunities," Sensors, vol. 14, no. 5, pp. 9153-9209, 2014.

[6] D. B. Arbia, M. M. Alam, R. Attia, and E. B. Hamida, "Data dissemination strategies for emerging wireless body-to-body networks based internet of humans," in proceedings of the Workshop on Advances in Body-Centric Wireless Communications and Networks and Their Applications (BCWNets 2015), in 11th IEEE WiMob Conference, Oct. 2015.

[7] G. C. Zongqing Lu and T. L. Porta, "Networking smartphones for disaster recovery," Proceedings of IEEE PerCom, 2016, 2016.

[8] L. E. Quispe and L. M. Galan, "Review: Behavior of ad hoc routing protocols, analyzed for emergency and rescue scenarios, on a real urban area," Expert Syst. Appl., vol. 41, no. 5, pp. 2565-2573, 2014.

[9] S. Saha, S. Nandi, P. S. Paul, V. K. Shah, A. Roy, and S. K. Das, "Designing delay constrained hybrid ad hoc network infrastructure for post-disaster communication," Ad Hoc Networks, vol. 25, pp. 406-429, 2015.

[10] A. Laha, X. Cao, W. Shen, X. Tian, and Y. Cheng, "An energy efficient routing protocol for device-to-device based multihop smartphone networks," in Communications (ICC), 2015 IEEE International Conference on, June 2015, pp. 5448-5453.

[11] J. P. P. Varun G. Menon and J. Priya, "Ensuring reliable communication in disaster recovery operations with reliable routing technique," Mobile Information Systems, p. 10, 2016.

[12] S. M. George, W. Zhou, H. Chenji, M. Won, Y. O. Lee, A. Pazarloglou, R. Stoleru, and P. Barooah, "Distressnet: a wireless ad hoc and sensor network architecture for situation management in disaster response," IEEE Communications Magazine, vol. 48, no. 3, pp. 128-136, March 2010.

[13] D. T. N. R. Group. (2015) Delay tolerant network 2. [Online]. Available: https://dtnrg.org/

[14] B. Karp and H. T. Kung, "Gpsr: Greedy perimeter stateless routing for wireless networks," in Proceedings of the 6th Annual International Conference on Mobile Computing and Networking, ser. MobiCom '00. New York, NY, USA: ACM, 2000, pp. 243-254. [Online]. Available: http://doi.acm.org/10.1145/345910.345953

[15] V. K. Sharma and D. S. S. Bhadauria, "Routing protocols for ad hoc mobile wireless networks: A comparative study," Wireless Communication, vol. 3, no. 11, 2011. [Online]. Available: http://www.ciitresearch.org/dl/index.php/wc/article/view/WC082011003

[16] T. Clausen, C. Dearlove, P. Jacquet, and U. Herberg, "Rfc7181: The optimized link state routing protocol version 2 (proposed standard)," Apr. 2014. [Online]. Available: http://www.rfc-editor.org/info/rfc7181

[17] C. Perkins, S. Ratliff, and D. J., "Dynamic manet on-demand (aodvv2) routing draft-ietf-manet-dymo-26," Apr. 2013. [Online]. Available: https://datatracker.ietf.org/doc/draft-ietf-manet-dymo/

[18] E. Ben Hamida and G. Chelius, "Investigating the impact of human activity on the performance of wireless networks: An experimental approach," in WoWMoM 2010 Conference, June 2010, pp. 1-8.

[19] N. Aschenbruck, R. Ernst, E. Gerhards-Padilla, and M. Schwamborn, "Bonnmotion: A mobility scenario generation and analysis tool," in proceedings of the 3rd International Conference on Simulation Tools and Techniques, 2010, pp. 51:1-51:10.

[20] "WSNET 3.0 Simulator," http://wsnet.gforge.inria.fr/, accessed: 201511-30.

[21] N. Aschenbruck, E. Gerhards-Padilla, M. Gerharz, M. Frank, and P. Martini, "Modelling mobility in disaster area scenarios," in Proceedings of the 10th ACM Symposium on Modeling, analysis, and simulation of wireless and mobile systems. ACM, 2007, pp. 4-12.

[22] M. M. Alam and E. B. Hamida, "Performance evaluation of ieee 802.15.6 mac for wearable body sensor networks using a space-time dependent radio link model," in 2014 IEEE/ACS 11th International Conference on Computer Systems and Applications (AICCSA), Nov 2014, pp. 441-448. 